\title{
A Signal Processing Method for Respiratory Rate Estimation through Photoplethysmography
}

\author{
Silvia Moreno $^{1 *}$, Andres Quintero-Parra ${ }^{2}$, Carlos Ochoa-Pertuz ${ }^{3}$, \\ Reynaldo Villarreal ${ }^{4}$ and Isaac Kuzmar ${ }^{5}$ \\ Universidad Simon Bolivar, Barranquilla, Colombia \\ ${ }^{1}$ smoreno12@unisimonbolivar.edu.co, ${ }^{2}$ aquintero30@unisimonbolivar.edu.co, \\ ${ }^{3}$ cochoa1@unisimonbolivar.edu.co, ${ }^{4}$ rvillarreal2@unisimonbolivar.edu.co, \\ ${ }^{5}$ isaac.kuzmar@unisimonbolivar.edu.co
}

\begin{abstract}
Monitoring of respiration is crucial for determining a patient's health status, specially previously and after an operation. However, many conventional methods are difficult to use in a spontaneously ventilating patient. This paper presents a method for estimating respiratory rate from the signal of a photoplethysmograph. This is a non-invasive sensor that can be used to obtain an estimation of beats per minute of a given patient by measuring light reflection on the patient's blood vessel and counting changes in blood flow. The PPG signal also offers information about respiration, so respiratory rate can be obtained through signal processing. The proposed method based on digital filtering was implemented in a wearable device and tested on 30 volunteers, and the results were compared with the ones measured by traditional ways. The results show that there is no statistically significant difference between the data measured by the device and the traditional method.
\end{abstract}

Keywords: biomedical signal processing, photoplethysmography, telemedicine, respiratory rate

\section{Introduction}

Vital signs reflect essential body functions, such as heartbeat, breathing rate, temperature, and blood pressure. A normal respiratory rate for an adult at rest is 12 to 18 breaths per minute. For an infant, a normal rate is up to 40 breaths per minute [1]. Continuous monitoring of respiration must be taken in order to explore a patient's health status, avoiding the risk of respiratory disorders as central or obstructive apnea, caused by opioid analgesics, neuromuscular blocking agents, and consequences after surgery [2]. Changes in the respiratory rate can also be a sensitive marker of impending respiratory dysfunction [3].

The measurement of respiratory rate can be done at a patient's home [4] or at a hospital. Some techniques include capnography and monitoring of transthoracic impedance that are difficult to use in a spontaneously ventilating patient. In order to overcome some of these problems a wearable non-invasive device with a photoplethysmograph has been developed that can be used to measure respiratory rate [5].

Photoplethysmography (PPG) is a technique used to obtain an estimation of beats per minute (BPM) of a given patient by measuring light reflection on the patient's blood vessel, and counting changes on blood flow [6]. This technique is widely used in pulse oximetry. However, the PPG signal can provide cardiac and respiratory rhythm information [7]. Through signal processing PPG can be used to estimate respiratory rate.

Received (November 21, 2017), Review Result (February 19, 2018), Accepted (February 26, 2018)

* Corresponding Author 
In this paper a method for measuring breath rate from the PPG signal is proposed. This method is applied by a device presented on [5] and tested on 30 patients. To validate the accuracy of the algorithm the results are compared with the ones obtained by physical observation.

\section{Previous Work}

There have been several authors that proposed methods and algorithms to extract respiratory rate from the PPG signal. Nilsson et al., [3] separate the signal in two minute blocks and apply Bessel bandpass filter between 0.13 and $0.48 \mathrm{~Hz}$, and then count the peaks of the signal to detect inspirations. Addison and Watson [8] apply wavelet decomposition to the PPG signal. Their method applies a secondary transform to the signal obtained from the original wavelet decomposition of the pulse band ridge. Other authors that apply wavelets are Lin et al., [9]. In this case the authors apply the Morlet Wavelet with the purpose of creating an efficient method that can be embedded in a microcontroller.

Another method based on filtering is the one proposed by Nakajima et al., [10]. In this method both heart and respiratory rate are measured simultaneously. First the heart and breathing signal are separated by filtering. The respiratory signal is filtered using low-pass filters with frequencies of $0.3,0.4$ and $0.55 \mathrm{~Hz}$. The cut-off frequency of the respiratory signal is selected automatically depending of the heart rate, and respiratory rate is estimated from the peak interval of the filtered signal. Other authors like Fleming and Tarassenko [11] propose a method that uses autoregressive modeling. In this method the signal is downsampled first in order to increase the angular resolution of the low frequency information. The authors claim that this method outperforms digital filtering and wavelet decomposition methods.

Independent Component Analysis (ICA) has also been used to extract respiratory activity from the PPG signal and estimate respiratory rate [12]. In the method proposed by Zhou et al., the latent variables of ICA take the form of the respiratory activity and the pulse wave. The mixture of these two variables was also measured, so the two channels of PPG signals (red and infrared) are considered. Finally, the ICA method is complemented by a JADE algorithm.

A different approach is one proposed by Garde et al., [13]. This method applies Correntropy Spectral Density (CSD), which is a generalization of the conventional power spectral density. CSD is based on the Fourier transform of the centered correntropy function. In the proposed approach first a window of 60 s or 120 s with $50 \%$ overlap is used to segment the signal, then CSD is applied and finally a low pass filter is used. Respiratory rate is estimated by detecting the maximum frequency peak within the respiratory frequency band.

Another method is the one proposed by Karlen et al., [14], called the Smart Fusion method. In this approach three variations, frequency, intensity and amplitude are obtained from the PPG signal applying an Incremental-Merge Segmentation algorithm and analyzed with Fast Fourier Transforms. The Smart Fusion method combines these three results and estimates respiratory rate. Shah et al., [15] propose a different approach. It is based in Autoregressive Modeling (AR), which estimates the power spectrum of a time series, constructs a median spectrum from multiple sets of AR model coefficients and then selects the dominant frequency in order to estimate respiratory rate. These authors also propose an algorithm to assess the PPG signal quality.

There have been methods based on the Fourier series. Madhav et al., [16] extract the respiratory activity from the PPG signal. The author applies adaptive Fourier coefficient estimator (AFCE) which models a quasi-periodic PPG signal as dynamic Fourier series. Fourier coefficients are estimated adaptively by a least mean squares 
(LMS) algorithm. This method is effective in extracting respiratory component and suppressing motion artifacts present in the PPG signals.

Other authors like Birrenkott et al., [17] have shown an interest in making more reliable and robust methods. These authors use Respiratory Quality Indices (RQIs) which assess the presence or absence of the respiratory modulations in the PPG and ECG signals. In this method first the respiratory modulations are extracted from the peaks and troughs of the signal, then these modulations are filtered with a Butterworth bandpass filter and downsampled. After this process the individual RQIs are calculated and then fused into a single RQI per modulation, which is used to estimate respiratory rate per time window.

Cicone and $\mathrm{Wu}$ [18] also focus on obtaining a more reliable method for extracting respiratory rate. In this case the authors apply theoretically solid techniques of nonlineartype time-frequency analyses, like the de-shape short time Fourier transform and the synchrosqueezing transform, which allows the estimation of respiratory rate with higher reliability,

Another method interested in robustness is the one proposed by Pimentel et al., [19]. In this method three Respiratory Induced time-series are extracted: Intensity Variation (RIIV), Amplitude Variation (RIAV) and Frequency Variation (RIFV). In order to identify artefactual and low-quality periods of the PPG waveform, a signal quality metric is used to assess the signal. If this metric is below a threshold, the window is discarded. This method estimates the respiratory rate by combining spectral estimates of the three pre-processed outputs (RIIV, RIAV, RIFV) using multiple Autoregressive Models (AR).

On the other hand, Orphanidou [20] proposes an algorithm for processing PPG signal that contain motion artefacts, which eliminates the data corruption caused by noise. The author applies Ensemble Empirical Mode Decomposition (EEMD), a popular noiseassisted data analysis method to achieve this goal. Another authors that apply EEMD are Motin et al., [21]. This method starts by applying Ensemble Empirical Mode Decomposition to decompose each segment of a PPG signal into a series of embedded Intrinsic Mode Functions (IMFs). In the second stage, the IMF containing artifacts is automatically identified and rejected. In the third stage, Principal Component Analysis (PCA) is applied and the first and second principal components are retained for extracting heart rate, respiratory rate and respiratory activity.

Zhang and Ding [22] propose a method that applies sparse signal reconstruction (SSR) based on orthogonal matching pursuit (OMP). This method first has a preprocessing stage where the signal is downsampled in order to improve efficiency. The Respiratory Rate Tracking stage of the method uses kurtosis of the SSR spectrum as a signal quality index and classifies the signal as good, moderate or poor. This quality index and the SSR spectrum are used to estimate respiratory rate.

Other authors apply Machine Learning techniques. Dubey et al., [23] develop a method based on Extreme Learning Machine (ELM) regression for estimating respiration rate. The features for this model are spectral kurtosis features, and the previously mentioned respiratory-induced features: amplitude, intensity and frequency variations. Table 1 shows a summary of these previous methods.

Most of these methods are computationally expensive and hard to apply in real-time measuring of breathing rate. In this paper we propose a method based on digital filtering that can be implemented with limited resources, like in a small wearable device, and presents good accuracy compared to commercial products. 


\section{Table 1. Summary of Methods for Respiratory Rate Estimation}

\begin{tabular}{|c|c|}
\hline Author & Technique \\
\hline Nilsson et al. & Digital Filtering \\
\hline Nakajima et al. & Digital Filtering \\
\hline Birrenkott et al. & Digital Filtering \\
\hline Addison and Watson & Wavelets \\
\hline Lin et al. & Wavelets \\
\hline Fleming and Tarassenko & Autoregressive Modeling \\
\hline Shah et al. & Autoregressive Modeling \\
\hline Pimentel et al. & Autoregressive Modeling \\
\hline Zhou et al. & Independent Component Analysis \\
\hline Garde et al. & Correntropy Spectral Density \\
\hline Karlen et al. & Fast Fourier Transforms \\
\hline Madhav et al. & Adaptive Fourier Coefficient \\
& Estimator \\
\hline Cicone and Wu & De-shape short time Fourier \\
& Transform \\
\hline Orphanidou & Ensemble Empirical Mode \\
& Decomposition \\
\hline Motin et al. & Ensemble Empirical Mode \\
& Decomposition \\
& Principal Component Analysis \\
\hline Zhang and Ding & Sparse Signal Reconstruction \\
\hline Dubey et al. & Extreme Learning Machine \\
\hline
\end{tabular}

\section{Materials and Methods}

\subsection{Method for Estimating Respiratory Rate}

In order to obtain respiratory rate a method based on the one proposed by Nilsson et al [3] was applied. The PPG signal is analyzed in two minute blocks. First a fourth order Bessel bandpass filter is applied to the signal with cut frequencies of 0.13 and $0.48 \mathrm{~Hz}$. Figure 1 shows a sample of the obtained PPG signal. Figure 2 presents a sample of the resulting signal after filtering.

Analyzing the filtered signal, the number of ascending peaks show patient's inspirations. Based on this data, a new procedure is applied to determine maximum and minimum peaks of the filtered signal. However, there may be small undulations in the filtered signal that do not represent inspirations, like the one highlighted on Figure 2, so a minimum peak height must be established. This value is calculated taking into account the mean height of maximum peaks, the peak's standard deviation and the total number of peaks. Finally, all maximum peaks that surpass the minimum height are counted, and the respiratory rate in breaths per minute is inferred. 


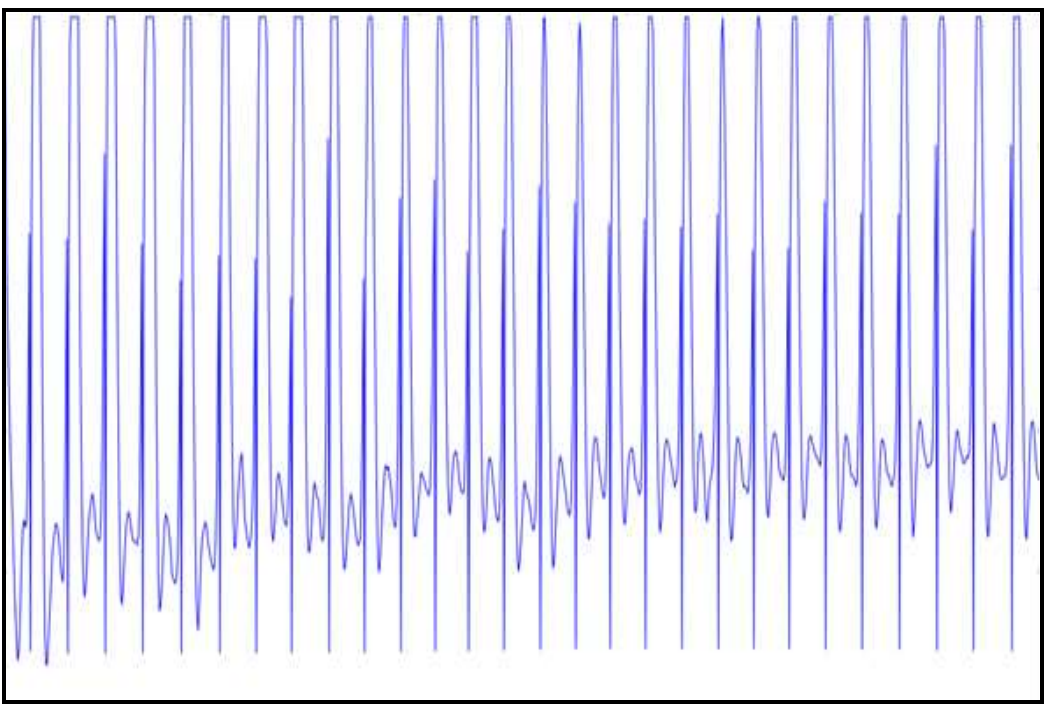

Figure 1. Sample of PPG Signal

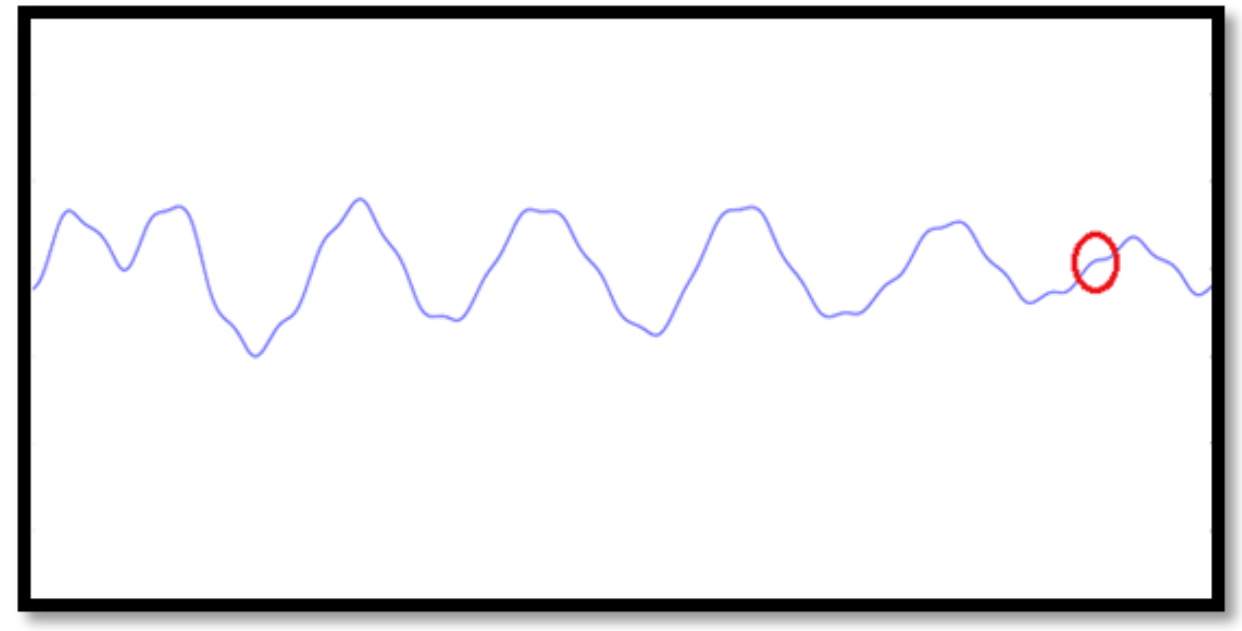

Figure 2. Sample of Filtered Signal

A Bessel filter is recommended because it has a response with a similar shape to the original signal, and it presents a flat frequency domain response, which is really useful for medical purposes, and it provides a better frequency response than Butterworth filters, due to its transfer function. Butterworth filters distorted the original PPG signals and provided wrong numbers of peaks, due to its flat frequency response, and its time domain response is unlike any PPG signal obtained.

The data set is taken at a $50 \mathrm{~Hz}$ sampling frequency, because, according to Nyquist theorem, it should be at least two times greater than its maximum, so any value from 25 $\mathrm{Hz}$ onwards could have been used, but $50 \mathrm{~Hz}$ provided enough information to obtain a correct estimate of breaths per minute. It was also used because of its low computational cost, the amount of data available on system's buffer and estimated battery life expectation.

\subsection{Implementation}

In order to validate the proposed method it was implemented on a wearable device described in [5]. This device was originally designed with the purpose of providing a 
cheap solution for measuring the patient's vital signs in the emergency room. Figure 3 presents a schematic of the device and it has the following components:

- Arduino: it is the device CPU. It receives the information from the sensors and transmits the data to a remote server via wireless connection. Signal processing can be done both by the arduino and the server.

- Temperature sensor: It measures the relative body temperature on a point of interest, which is the wrist for these tests, and sends the data to the processor.

- Photophletysmograph: this is the key sensor for obtaining pulse and respiratory rate. It is an optic instrument that allows detection of blood volume in micro vascular tissue by taking measures through the skin surface and detecting changes in light absorption. This sensor is placed on the person's fingertip and it transmits the PPG signal that is used to calculate heart and respiratory rate.

- Wireless transceiver: the Wifi module transmits a data vector with the processed information from the sensors to the server, where it can be used by an information system for monitoring vital signs.

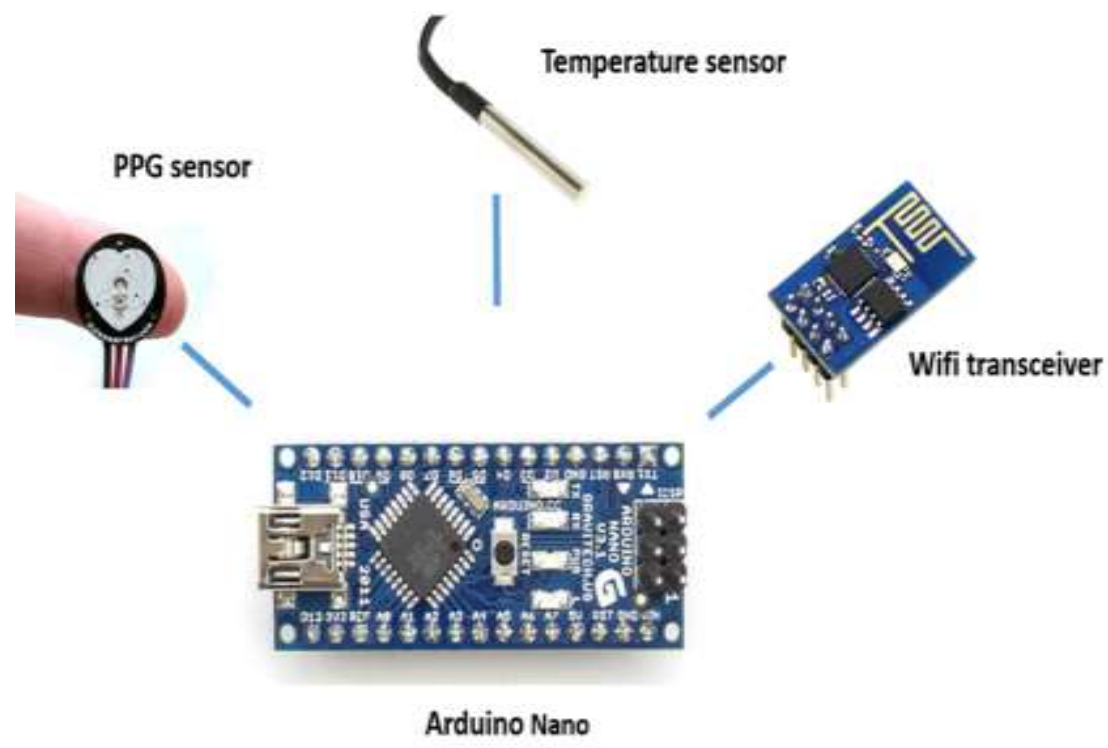

Figure 3. Schematic of the Device

The proposed method was implemented in the device using the arduino programming language. It was also implemented in a remote processing version on python 3.4.

\subsection{Validation}

The device implementing the proposed method was tested on 30 spontaneously breathing healthy adult volunteers. Results were compared with the respiratory rate obtained by traditional methods measured by a health-trained person and every sample was doubled checked to minimize errors. All people were aged between 30 and 40 years old, and reported no health disease. The study was conducted according to Helsinki Rules pertaining to all patients informed consent.

For the validation process the following procedure was applied: first the device was turned on and attached to the subject. Due to the adjustment time needed by the sensors, two minutes passed before taking any measurements. During the test, the respiratory rate was measured simultaneously by the device and physical observation. 


\section{Results}

The validation tests were performed as described above. Table 2 shows the respiratory rate measured in breaths per minute both by our device and by physical observation. The results were analyzed with the software IBM SPSS Statistics version 23. In order to determine if there is a significant difference between the results obtained by the device and traditional measurements, the Wilcoxon signed rank test for related samples was applied. This test was selected because it did not require the data to be normally distributed. The result of the analysis performed by SPSS is shown on Figure 4.

Table 2. Respiratory Rate Measurements in Breaths per Minute

\begin{tabular}{|l|l|c|c|}
\hline & & \multicolumn{2}{|c|}{ Breaths per minute } \\
\hline ID & Gender & Device & Physical Observation \\
\hline 1 & M & 16 & 16 \\
\hline 2 & M & 16 & 16 \\
\hline 3 & F & 17 & 16 \\
\hline 4 & F & 16 & 16 \\
\hline 5 & F & 12 & 16 \\
\hline 6 & M & 12 & 12 \\
\hline 7 & F & 14 & 24 \\
\hline 8 & M & 13 & 12 \\
\hline 9 & F & 13 & 12 \\
\hline 10 & M & 16 & 16 \\
\hline 11 & F & 16 & 24 \\
\hline 12 & M & 15 & 16 \\
\hline 13 & F & 11 & 20 \\
\hline 14 & M & 13 & 12 \\
\hline 15 & M & 11 & 12 \\
\hline 16 & M & 16 & 16 \\
\hline 17 & M & 16 & 16 \\
\hline 18 & M & 16 & 16 \\
\hline 19 & F & 14 & 16 \\
\hline 20 & F & 11 & 12 \\
\hline 21 & M & 16 & 16 \\
\hline 22 & M & 22 & 20 \\
\hline 23 & F & 18 & 18 \\
\hline 24 & F & 20 & 20 \\
\hline 25 & M & 16 & 16 \\
\hline 26 & M & 12 & 12 \\
\hline 27 & M & 16 & 20 \\
\hline 28 & M & 20 & 20 \\
\hline 29 & F & 20 & \\
\hline 30 & F & 20 & 20 \\
\hline & & & 16 \\
\hline
\end{tabular}


Hypothesis Test Summary

\begin{tabular}{|c|c|c|c|}
\hline Null Hypothesis & Test & Sig. & Decision \\
\hline $\begin{array}{l}1 \text { The median of differences between } \\
\text { DEVICE and PHYS_OBS equals } 0 \text {. }\end{array}$ & $\begin{array}{l}\text { Related- } \\
\text { Samples } \\
\text { Wilcoxon } \\
\text { Signed Rank } \\
\text { Tesst }\end{array}$ & ,135 & $\begin{array}{l}\text { Retain the } \\
\text { null } \\
\text { hypothesis }\end{array}$ \\
\hline
\end{tabular}

Asymptotic significances are displayed. The significance level is ,05.

Figure 4. Results of Wilcoxon Signed Ranked Test

The test estimated a P-value of 0.135 , which is higher than 0.05 . This indicates that there is not enough statistical evidence to reject the null hypothesis with a $95 \%$ of confidence, which states that the two measurements (device and physical observation) are not significantly different. This result supports that the implementation of the proposed method is accurate enough compared with traditional methods, and can be easily implemented in a wearable device. The method shows a mean error of 1.4 breaths per minute, this is an acceptable performance for most medical applications. Figure 5 shows a boxplot of the difference between the measures obtained by the device and the physical observations.

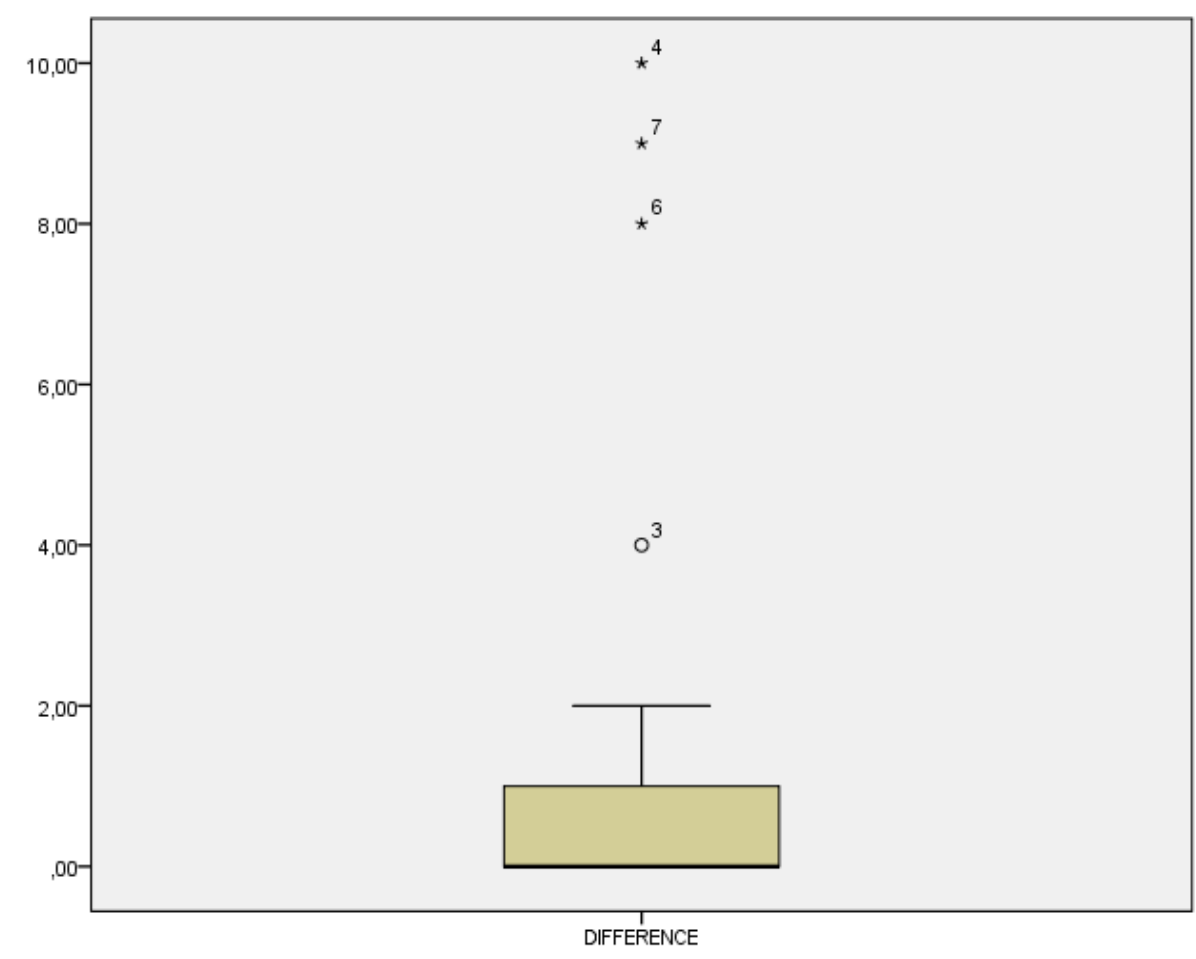

Figure 5. Boxplot of Difference between the Two Measurements 


\section{Conclusions}

A method for estimating respiratory rate from the photoplethysmograph signal has been presented. This method is not computationally expensive and shows an adequate accuracy. In the validation tests there was no statistically significant difference between the measures obtained by the device with the implementation of the method and the ones obtained by traditional methods.

The possibility of measuring the breath rate in one skin sensor makes PPG attractive as a method for pre or postoperative monitoring of vital signs, and for many other medical applications. For future work a more exhaustive validation should be made. It is necessary to expand the sample of volunteers, from all ranks of ages, healthy and sick, and develop new studies in order to share new results with the international scientific community. Also the proposed method will be tested with well-known benchmark datasets, such as CapnoBase [24] and its performance will be compared with other recent algorithms.

Another focus of future work will be improving the algorithms robustness. The method should be able to detect and discard motion artifacts and low-quality samples of the signal automatically, so the accuracy of the measures does not get compromised if these probable eventualities occur.

\section{References}

[1] L. Goldman, "Goldman-Cecil medicine", Philadelphia, PA: Elsevier/Saunders, (2016).

[2] Z. Sun, "Postoperative Hypoxemia Is Common and Persistent: A Prospective Blinded Observational Study", Anesth. Analg., vol. 121, no. 3, (2015) September, pp. 709-715.

[3] L. Nilsson, A. Johansson, and S. Kalman, "Monitoring of respiratory rate in postoperative care using a new photoplethysmographic technique", PubMed Commons, vol. 16, no. 4, (2001), pp. 309-315.

[4] N. Patwari, L. Brewer, Q. Tate, O. Kaltiokallio and M. Bocca, "Breathfinding: A Wireless Network That Monitors and Locates Breathing in a Home”, IEEE J. Sel. Top. Signal Process., vol. 8, no. 1, (2014) February, pp. 30-42.

[5] S. Moreno, A. Quintero, C. Ochoa, M. Bonfante, R. Villareal and J. Pestana, "Remote monitoring system of vital signs for triage and detection of anomalous patient states in the emergency room", 2016 21 st Symp. Signal Process. Images Artif. Vision, STSIVA 2016, (2016), pp. 1-5.

[6] A. Schäfer and J. Vagedes, "How accurate is pulse rate variability as an estimate of heart rate variability?", Int. J. Cardiol., vol. 166, no. 1, (2018) January, pp. 15-29.

[7] L. Nilsson, A. Johansson, J. Svanerudh and S. Kalman, "Is the respiratory component of the photoplethysmographic signal of venous origin?”, Med. Biol. Eng. Comput., vol. 37, (1999), pp. 912913.

[8] P. S. Addison and J. N. Watson, "Secondary wavelet feature decoupling (SWFD) and its use in detecting patient respiration from the photoplethysmogram", Proceedings of the 25th Annual International Conference of the IEEE Engineering in Medicine and Biology Society (IEEE Cat. No.03CH37439), 2003, vol. 3, p. 2602-2605.

[9] Y. Der Lin, Y. H. Chien and Y. S. Chen, "Wavelet-based embedded algorithm for respiratory rate estimation from PPG signal”, Biomed. Signal Process. Control, vol. 36, (2017), pp. 138-145.

[10] K. Nakajima, T. Tamura and H. Miike, "Monitoring of heart and respiratory rates by photoplethysmography using a digital filtering technique", Med. Eng. Phys., vol. 18, no. 5, (1996), pp. 365-372.

[11] S. G. Fleming and L. Tarassenko, "A Comparison of Signal Processing Techniques for the Extraction of Breathing Rate from the Photoplethysmogram", Int. J. Biol. Life Sci., vol. 2, no. 4, (2006), pp. 233-237.

[12] Y. Zhou, Y. Zheng, C. Wang and J. Yuan, "Extraction of respiratory activity from photoplethysmographic signals based on an independent component analysis technique: Preliminary report", Instrum. Sci. Technol., vol. 34, no. 5, (2006), pp. 537-545.

[13] A. Garde, W. Karlen, J. M. Ansermino and G. A. Dumont, "Estimating respiratory and heart rates from the correntropy spectral density of the photoplethysmogram", PLoS One, vol. 9, no. 1, (2014).

[14] W. Karlen, S. Raman, J. M. Ansermino and G. A. Dumont, "Multiparameter respiratory rate estimation from the photoplethysmogram", IEEE Trans. Biomed. Eng., vol. 60, no. 7, (2013), pp. 1946-1953.

[15] S. A. Shah, S. Fleming, M. Thompson and L. Tarassenko, "Respiratory rate estimation during triage of children in hospitals", J. Med. Eng. Technol., vol. 39, no. 8, (2015), pp. 514-524.

[16] K. V. Madhav, E. H. Krishna and K. A. Reddy, "Extraction of respiratory activity from pulse oximeter's PPG signals using MSICA”, Proc. 2016 IEEE Int. Conf. Wirel. Commun. Signal Process. Networking, WiSPNET 2016, (2016), pp. 823-827.

[17] D. Birrenkott, M. A. F. Pimentel, P. J. Watkinson and D. A. Clifton, "A Robust Fusion Model for 
Estimating Respiratory Rate from Photoplethysmography and Electrocardiography”, IEEE Trans. Biomed. Eng., no. c, (2017), pp. 1-9.

[18] A. Cicone and H. T. Wu, "How nonlinear-type time-frequency analysis can help in sensing instantaneous heart rate and instantaneous respiratory rate from photoplethysmography in a reliable way", Front. Physiol., vol. 8, no. SEP, (2017), pp. 1-17.

[19] M. A. F. Pimentel, "Toward a robust estimation of respiratory rate from pulse oximeters", IEEE Trans. Biomed. Eng., vol. 64, no. 8, (2017), pp. 1914-1923.

[20] C. Orphanidou, "Derivation of respiration rate from ambulatory ECG and PPG using Ensemble Empirical Mode Decomposition: Comparison and fusion”, Comput. Biol. Med., vol. 81, (2017), pp. 4554.

[21] M. A. Motin, C. K. Karmakar and M. Palaniswami, "An EEMD-PCA approach to extract heart rate, respiratory rate and respiratory activity from PPG signal”, Proc. Annu. Int. Conf. IEEE Eng. Med. Biol. Soc. EMBS, vol. 2016-October, no. 0, (2016), pp. 3817-3820.

[22] X. Zhang and Q. Ding, "Fast respiratory rate estimation from PPG signal using sparse signal reconstruction based on orthogonal matching pursuit”, 2016 50th Asilomar Conf. Signals, Syst. Comput., (2016), pp. 1631-5.

[23] H. Dubey, N. Constant and K. Mankodiya, "RESPIRE: A Spectral Kurtosis-Based Method to Extract Respiration Rate from Wearable PPG Signals", Proc. - 2017 IEEE 2nd Int. Conf. Connect. Heal. Appl. Syst. Eng. Technol. CHASE 2017, (2017), pp. 84-89.

[24] Electrical and Computer Engineering in Medicine, "CapnoBase," 2010. [Online]. Available: http://www.capnobase.org/. [Accessed: 23-Feb-2018]. 